\title{
By Magri's Theorem, Self-Dual Gravity is Completely Integrable ${ }^{\star}$
}

Yavuz NUTKU

Feza Gürsey Institute, P.O.Box 6, Çengelköy, Istanbul, 81220 Turkey

E-mail: nutku@gursey.gov.tr, notcoo@yahoo.com

Received September 08, 2006, in final form February 08, 2007; Published online February 27, 2007

Original article is available at http://www.emis.de/journals/SIGMA/2007/034/

\begin{abstract}
By Magri's theorem the bi-Hamiltonian structure of Plebanski's second heavenly equation proves that (anti)-self-dual gravity is a completely integrable system in four dimensions.
\end{abstract}

Key words: self-dual gravity; Plebanski equation; Magri's theorem

2000 Mathematics Subject Classification: 83C20; 83C15; 37K10

\section{Introduction}

The purpose of this work is to present a recent result published in my paper with Neyzi and Sheftel [1], namely, the bi-Hamiltonian structure [2,3] of Plebanski's second heavenly equation [4].

Perhaps I may be allowed to start with a personal remark by way of introduction. The first and the last time, before here in Coimbra, that I had the pleasure of talking to Professor Magri was close to 20 years ago. I was just coming out of mid-life crisis precipitated by having proved a very beautiful result in general relativity [5] that I thought I could never equal again and also divorce with very young children. I felt I had to change everything. So I looked around and found shocks. Certainly what I thought was the best developed subject in physics was the symplectic structure underlying Newtonian mechanics. The great works of Poisson, Hamilton, Jacobi, Liouville and Darboux two centuries after Newton had made it a perfect gem. In particular the theorem of Darboux stating that the symplectic 2-form can be put into the form given in the poster for this conference was the final nail on the coffin. Mind you this is not a local theorem but, barring singularities, holds in all of phase space. All this had made the theory of symplectic structure a fossilized subject fit for Bourbaki!

Yet along came Nambu [6] and Magri [2,3] who changed all that. In particular it was Magri who proved a very beautiful and powerful theorem that an evolutionary system may admit more than one Hamiltonian structure and was therefore completely integrable. I found this most fascinating and started working on gas dynamics which turned out to admit tri-Hamiltonian structure [7] and later on was shown to admit one more [8]. However, nowadays most of the people in integrable systems refer to [8] instead of [7] where the original results first appeared. I find it ironic that the people who originally shrugged off my results in [7] as being a trivial 2-component system in $1+1$ dimensions now use it as the cornerstone of their work without supplying the correct reference.

So I was full of enthusiasm meeting Magri 20 years ago and wanted to talk to him about his theorem. However, he just shrugged off and said that it was an old result. Now the theorem of Pythagoras came two and a half millennia ago, have you ever seen any reference to it as being

${ }^{\star}$ This paper is a contribution to the Proceedings of the Workshop on Geometric Aspects of Integrable Systems (July 17-19, 2006, University of Coimbra, Portugal). The full collection is available at http://www.emis.de/journals/SIGMA/Coimbra2006.html 
old! Great theorems are never old and Magri's theorem is a great one. On the other hand its author is privileged to call it anything he likes.

Now I shall present you what I believe is the most important application of Magri's theorem.

\section{Self-dual Gravity}

There are scalar-valued equations governing Riemannian metrics with (anti)-self-dual Riemann 2 -form. By Bianchi's first identity this implies Ricci-flatness. First we have the complex MongeAmpère equation for Kähler metrics [9]. This has the form of the Monge-Ampère determinant set equal to a constant. Next we have the second heavenly equation of Plebanski [4]. They are related by a Legendre transformation which in the context of Riemannian geometry is a coordinate transformation together with a redefinition of the Kähler potential. The second heavenly equation of Plebanski, hereafter denoted as $P_{2}$,

$$
u_{t t} u_{x x}-u_{t x}^{2}+u_{x z}+u_{t y}=0,
$$

is close to the real Monge-Ampère equation that has rich Hamiltonian structure [10] and will be the subject of our investigation.

\section{$3 \quad$ First order form of $\boldsymbol{P}_{2}$}

The second heavenly equation is a second order partial differential equation. There is confusion in the literature as to whether or not the independent variables in $P_{2}$ are complex, as Plebanski originally presented, or real. We shall take them to be real which leads to Euclidean signature for the metric. In order to discuss its Hamiltonian structure we shall single out an independent variable, $t$, in (1) to play the role of "time" and express $P_{2}$ as a pair of first order nonlinear evolution equations. Thus we introduce an auxiliary variable $q$ whereby (1) assumes the form

$$
u_{t}=q, \quad q_{t}=\frac{1}{u_{x x}}\left(q_{x}^{2}-q_{y}-u_{x z}\right) \equiv Q
$$

of a first order system. For the sake of brevity we shall henceforth refer to $(2)$ as the $P_{2}$-system. It is worth noting that this split of (1) into the system (2) is not unique, here we are using the most straight-forward choice. Now the vector field

$$
\mathbf{X}=q \frac{\partial}{\partial u}+Q \frac{\partial}{\partial q}
$$

defines the flow. In the discussion of the Hamiltonian structure of this system we shall use matrix notation with $u^{i}(i=1,2), u^{1}=u, u^{2}=q$ running over the dependent variables.

The equations of motion (2) are to be cast into the form of Hamilton's equations in two different ways according to the recursion relation of Magri

$$
u_{t}^{i}=\mathbf{X}\left(u^{i}\right)=J_{0}^{i k} \delta_{k} H_{1}=J_{1}^{i k} \delta_{k} H_{0},
$$

where $\delta_{k}$ denotes variational derivative of the Hamiltonian functional $H_{i}=\int_{\infty}^{\infty} \mathcal{H}_{i} d x d y d z$ with respect to $u^{k}$.

\section{Lagrangian and Dirac's theory of constraints}

In order to arrive at the first Hamiltonian structure of the $P_{2}$-system, we start with its Lagrangian

$$
\mathcal{L}=q u_{t} u_{x x}+\frac{1}{2} u_{t} u_{y}-\frac{1}{2} q^{2} u_{x x}+\frac{1}{2} u_{x} u_{z}
$$


which is degenerate because its Hessian vanishes. Thus we need to apply Dirac's theory of constraints [11] in order to cast it into Hamiltonian form. We define canonical momenta which satisfy canonical Poisson brackets in the usual way. But we find that they cannot be inverted for the velocities which must therefore be imposed as constraints

$$
\phi_{u}=\pi_{u}-\left(q u_{x x}+\frac{1}{2} u_{y}\right), \quad \phi_{q}=\pi_{q}
$$

and calculate the Poisson bracket of the constraints

$$
K_{i k}=\left[\phi_{i}(x, y, z), \phi_{k}\left(x^{\prime}, y^{\prime}, z^{\prime}\right)\right]=K_{i k}(x, y, z) \delta\left(x-x^{\prime}\right) \delta\left(y-y^{\prime}\right) \delta\left(z-z^{\prime}\right)
$$

which plays an important role. The symplectic 2 -form is obtained by integrating the density

$$
\omega=\frac{1}{2} d u^{i} \wedge K_{i j} d u^{j}
$$

and it is straightforward to verify that the density of symplectic 2-form is determined by

$$
K=\left(\begin{array}{cc}
q_{x} D_{x}+D_{x} q_{x}-D_{y} & -u_{x x} \\
u_{x x} & 0
\end{array}\right)
$$

which is a first order local operator. Thus we find the symplectic 2-form

$$
\omega=q_{x} d u \wedge d u_{x}-u_{x x} d u \wedge d q-\frac{1}{2} d u \wedge d u_{y}
$$

which, up to a divergence, can be directly verified to be a closed 2-form.

The statement of the symplectic structure of the equations of motion (2) consists of

$$
i_{X} \omega=d H
$$

which is obtained by the contraction of the closed symplectic 2 -form $\omega$ with the vector field $\mathbf{X}$ (3) defining the flow.

\section{First Hamiltonian structure}

The first Hamiltonian operator for $P_{2}$-system is obtained by inverting (4) to arrive at the Dirac bracket. This is given by

$$
J_{0}=\left(\begin{array}{cc}
0 & \frac{1}{u_{x x}} \\
-\frac{1}{u_{x x}} & \frac{q_{x}}{u_{x x}^{2}} D_{x}+D_{x} \frac{q_{x}}{u_{x x}^{2}}-\frac{1}{u_{x x}} D_{y} \frac{1}{u_{x x}}
\end{array}\right)
$$

which, apart from the $D_{y}$ term in its last entry, is simply the first Hamiltonian operator for the real Monge-Ampère equation [10]. It can be directly verified that

$$
\mathcal{H}_{1}=\frac{1}{2} q^{2} u_{x x}-\frac{1}{2} u_{x} u_{z}
$$

is conserved for the flow (3) and this is the first Hamiltonian density appropriate to the operator $(6)$.

The proof of the Jacobi identities for the Hamiltonian operator (6) is straight-forward but rather lengthy. A shorter proof follows from the observation that (6) is the inverse of (4) which leads to the closed 2-form (5). 


\section{Recursion operator}

We shall use the recursion operator in order to arrive at the second Hamiltonian structure of the $P_{2}$-system. However, it is worth recalling that the recursion operator originates in symmetry analysis. So we start with the equation determining the symmetries of the $P_{2}$-system and introduce two components for symmetry characteristics

$$
u_{\tau}=\varphi, \quad q_{\tau}=\psi, \quad \Phi \equiv\left(\begin{array}{c}
\varphi \\
\psi
\end{array}\right)
$$

while from the Frechét derivative of the flow we find

$$
\mathcal{A}=\left(\begin{array}{cc}
D_{t} & -1 \\
\frac{Q}{u_{x x}} D_{x}^{2}+\frac{1}{u_{x x}} D_{x} D_{z} & D_{t}-\frac{2 q_{x}}{u_{x x}} D_{x}+\frac{1}{u_{x x}} D_{y}
\end{array}\right)
$$

so that the equation determining the symmetries of second heavenly system is given by

$$
\mathcal{A}(\Phi)=0 .
$$

This determining equation has the divergence form

$$
\left(u_{x x} \psi-q_{x} \varphi_{x}+\varphi_{y}\right)_{t}+\left(q_{t} \varphi_{x}-q_{x} \psi+\varphi_{z}\right)_{x}=0
$$

which implies the local existence of the potential variable $\tilde{\varphi}$ such that

$$
\tilde{\varphi}_{t}=q_{t} \varphi_{x}-q_{x} \psi+\varphi_{z}, \quad \tilde{\varphi}_{x}=-\left(u_{x x} \psi-q_{x} \varphi_{x}+\varphi_{y}\right)
$$

that satisfies the same determining equation for symmetries of (1) and therefore is a "partner symmetry" [12] for (7). In the two-component form we define the second component of this new symmetry similar to the definition of $\psi$ as $\tilde{\psi}=\tilde{\varphi}_{t}$. Then the two-component vector

$$
\tilde{\Phi}=\left(\begin{array}{c}
\tilde{\varphi} \\
\tilde{\psi}
\end{array}\right)
$$

satisfies the determining equation for symmetries in the form (9) and hence is a symmetry characteristic of the system (2) provided the vector (7) is also a symmetry characteristic. Thus (10) become the recursion relation for symmetries in the two-component form

$$
\tilde{\Phi}=\mathcal{R}(\Phi)
$$

with the recursion operator given by

$$
\mathcal{R}=\left(\begin{array}{cc}
D_{x}^{-1}\left(q_{x} D_{x}-D_{y}\right) & -D_{x}^{-1} u_{x x} \\
Q D_{x}+D_{z} & -q_{x}
\end{array}\right),
$$

where $D_{x}^{-1}$ is the inverse of $D_{x}$. See [13] for the definition and properties of this operator, in particular,

$$
D_{x}^{-1} f=\frac{1}{2}\left(\int_{-\infty}^{x}-\int_{x}^{\infty}\right) f(\xi) d \xi
$$

and the integrals are taken in the principal value sense. 
The commutator of the recursion operator (11) and the operator determining symmetries (8) has the form

$$
[\mathcal{R}, \mathcal{A}]=\left(\begin{array}{cc}
D_{x}^{-1}\left(q_{t}-Q\right)_{x x}-\left(q_{t}-Q\right)_{x} & D_{x}^{-1}\left(u_{t}-q\right)_{x x} \\
\left\{\frac{Q}{u_{x x}}\left(u_{t}-Q\right)_{x x}+\left(D_{y}-\frac{2 q_{x}}{u_{x x}}\right)\left(q_{t}-Q\right)_{x}\right\} D_{x} & \left(q_{t}-Q\right)_{x}
\end{array}\right)
$$

and as a consequence, the operators $\mathcal{R}$ and $\mathcal{A}$ commute

$$
[\mathcal{R}, \mathcal{A}]=0
$$

by virtue of the $P_{2}$-system (2). Moreover, $\mathcal{R}$ and $\mathcal{A}$ form a Lax pair for the second heavenly system.

\section{Second Hamiltonian structure}

The second Hamiltonian operator $J_{1}$ is obtained by applying the recursion operator (11) to the first Hamiltonian operator $J_{1}=\mathcal{R} J_{0}$. We find

$$
J_{1}=\left(\begin{array}{cc}
D_{x}^{-1} & -\frac{q_{x}}{u_{x x}} \\
\frac{q_{x}}{u_{x x}} & -\frac{1}{2}\left(Q D_{x} \frac{1}{u_{x x}}+\frac{1}{u_{x x}} D_{x} Q\right) \\
& +\frac{1}{2}\left(\frac{q_{x}}{u_{x x}} D_{y} \frac{1}{u_{x x}}+\frac{1}{u_{x x}} D_{y} \frac{q_{x}}{u_{x x}}+\frac{1}{u_{x x}} D_{z}+D_{z} \frac{1}{u_{x x}}\right)
\end{array}\right)
$$

which is manifestly skew. The proof of the Jacobi identity is again straight-forward and lengthy.

The Hamiltonian operators (6) and (12) form a Poisson pencil, that is, every linear combination $a J_{0}+b J_{1}$ of these two Hamiltonian operators with constant coefficients $a$ and $b$ satisfies the Jacobi identities.

\section{Conclusion}

The second heavenly equation of Plebanski which determines Riemannian metrics with (anti)self-dual curvature 2-form implying Ricci-flatness admits bi-Hamiltonian structure. Therefore by Magri's theorem self-dual gravity is a completely integrable system in 4 dimensions.

\section{References}

[1] Neyzi F., Nutku Y., Sheftel M.B., Multi-Hamiltonian structure of Plebanski's second heavenly equation, J. Phys. A: Math. Gen. 38 (2005), 8473-8485, nlin.SI/0505030.

[2] Magri F., A simple model of the integrable Hamiltonian equation, J. Math. Phys. 19 (1978), 1156-1162.

[3] Magri F., A geometrical approach to the nonlinear solvable equations, in Nonlinear Evolution Equations and Dynamical Systems, Editors M. Boiti, F. Pempinelli and G. Soliani, Lecture Notes in Phys., Vol. 120, Springer, New York, 1980, 233-263.

[4] Plebanski J.F., Some solutions of complex Einstein equations, J. Math. Phys. 16 (1975), 2395-2402.

[5] Nutku Y., Halil M., Colliding impulsive gravitational waves, Phys. Rev. Lett. 39 (1977), 1379-1382.

[6] Nambu Y., Generalized Hamiltonian dynamics, Phys. Rev. D 7 (1973), 2405-2412.

[7] Nutku Y., On a new class of completely integrable nonlinear wave equations. II. Multi-Hamiltonian structure, J. Math. Phys. 28 (1987), 2579-2585. 
[8] Olver P.J., Nutku Y., Hamiltonian structures for systems of hyperbolic conservation laws, J. Math. Phys. 29 (1988), 1610-1619.

[9] Calabi E., The space of Kähler metrics, in Proceedings of the International Congress of Mathematicians (1954, Amsterdam), Vol. 2, North-Holland, Amsterdam, 1956, 206-207.

[10] Nutku Y., Hamiltonian structure of real Monge-Ampère equations, J. Phys. A: Math. Gen. 29 (1996), 3257-3280, solv-int/9812023.

[11] Dirac P.A.M., Lectures on quantum mechanics, Belfer Graduate School of Science Monographs Series 2, New York, 1964.

[12] Malykh A.A., Nutku Y., Sheftel M.B., Partner symmetries of the complex Monge-Ampère equation yield hyper-Kahler metrics without continuous symmetries, J. Phys. A: Math. Gen. 36 (2003), 10023-10037, math-ph/0403020.

[13] Santini P.M., Fokas A.S., Recursion operators and bi-Hamiltonian structures in multidimensions. I, Comm. Math. Phys. 115 (1988), 375-419. 